\title{
Correlation between cerebrospinal fluid and plasma neurofilament light protein in treated HIV infection: results from the COBRA study
}

\author{
Jasmini Alagaratnam ${ }^{1,2}$ (D) Davide De Francesco ${ }^{3} \cdot$ Henrik Zetterberg $4,5,6,7$. Amanda Heslegrave ${ }^{4}$ Jamie Toombs ${ }^{4}$. \\ Neeltje A. Kootstra ${ }^{8}$. Jonathan Underwood ${ }^{1,9,10} \cdot$ Magnus Gisslen $^{11,12}$ • Peter Reiss ${ }^{13,14,15} \cdot$ Sarah Fidler $^{1,2}$. \\ Caroline A. Sabin ${ }^{3}$ - Alan Winston ${ }^{1,2}$
}

Received: 17 July 2020 / Revised: 24 March 2021 / Accepted: 27 October 2021 / Published online: 7 December 2021

(c) The Author(s) 2021

\begin{abstract}
Cerebrospinal fluid (CSF) neurofilament light protein (NfL) is a marker of central nervous system neuro-axonal injury. A novel, ultra-sensitive assay can determine plasma NfL. In untreated people-with-HIV (PWH), CSF and plasma NfL are strongly correlated. We aimed to assess this correlation in PWH on suppressive antiretroviral treatment (ART) and lifestylesimilar HIV-negative individuals enrolled into the COmorBidity in Relation to AIDS (COBRA) study. Differences in paired CSF (sandwich ELISA, UmanDiagnostics) and plasma (Simoa digital immunoassay, Quanterix ${ }^{\mathrm{TM}}$ ) NfL between PWH and HIV-negative participants were tested using Wilcoxon's test; associations were assessed using Pearson's correlation. CSF and plasma NfL, standardised to $Z$-scores, were included as dependent variables in linear regression models to identify factors independently associated with values in PWH and HIV-negative participants. Overall, $132 \mathrm{PWH}$ (all with plasma HIV RNA $<50$ copies $/ \mathrm{mL}$ ) and $79 \mathrm{HIV}$-negative participants were included. Neither CSF (median $570 \mathrm{vs} 568 \mathrm{pg} / \mathrm{mL}, p=0.37$ ) nor plasma (median $10.7 \mathrm{vs} 9.9 \mathrm{pg} / \mathrm{mL}, p=0.15$ ) NfL differed significantly between PWH and HIV-negative participants, respectively. CSF and plasma NfL correlated moderately, with no significant difference by HIV status (PWH: rho $=0.52$; HIV-negative participants: rho $=0.47, p$ (interaction) $=0.63$ ). In multivariable regression analysis, higher CSF NfL $Z$-score was statistically significantly associated with older age and higher CSF protein, and higher plasma NfL Z-score with older age, higher serum creatinine and lower bodyweight. In conclusion, in PWH on ART, the correlation between CSF and plasma NfL is moderate and similar to that observed in lifestyle-similar HIV-negative individuals. Consideration of renal function and bodyweight may be required when utilising plasma NfL.
\end{abstract}

Keywords HIV $\cdot$ HIV-1 Central nervous system diseases $\cdot$ Axons $\cdot$ Neurofilament proteins

\section{Introduction}

Cerebrospinal fluid (CSF) neurofilament light chain protein (NfL) is well-established as a sensitive biomarker of active central nervous system (CNS) neuro-axonal injury (Gaetani et al. 2019; Khalil et al. 2018; Olsson et al. 2019). The neurofilaments form a major structural component of myelinated axons and maintain the structural and functional integrity of axons (Gaetani et al. 2019). Injured axons leak neurofilament protein into the CSF, proportional to the severity of the injury(Gaetani et al. 2019). Elevated CSF NfL concentrations are well-documented in many neurological

Jasmini Alagaratnam

j.alagaratnam@nhs.net

Extended author information available on the last page of the article disorders (Bridel et al. 2019; Gaetani et al. 2019; Khalil et al. 2018; Zetterberg 2016). In HIV-disease, CSF NfL is significantly elevated in individuals with HIV-associated dementia (Abdulle et al. 2007; Gisslén et al. 2007; Krut et al. 2014; Yilmaz et al. 2017), but frequently near-normalise following antiretroviral treatment (ART) (Abdulle et al. 2007; Krut et al. 2014; Mellgren et al. 2007). Elevated CSF NfL concentrations can also be detected in neuroasymptomatic people with HIV (PWH) not on ART, especially in those with low $\mathrm{CD}^{+}{ }^{+}$counts (Krut et al. 2014; Peterson et al. 2014).

The invasive nature of CSF collection via lumbar puncture significantly limits its widespread use. A novel ultrasensitive assay can determine plasma NfL (Gisslén et al. 2015; Hendricks et al. 2019), which removes the barriers faced by CSF collection and allows more frequent measurement. 
Early studies suggest that CSF strongly correlates with blood NfL (Disanto et al. 2017; Hansson et al. 2017; Kuhle et al. 2016; Marques et al. 2019; Novakova et al. 2017), including in a cohort of mainly untreated PWH (Gisslén et al. 2015). However, whether this strong correlation persists in PWH on virally suppressive ART is unknown. Once $\mathrm{NfL}$ is released from CNS neuro-axonal units, it reaches the interstitial fluid which communicates freely with CSF and blood. While it could be postulated that an impaired blood-brain barrier (from untreated HIV-disease or alcohol and recreational drug use) may be associated with higher transfer of NfL from the CSF into the bloodstream, several studies have demonstrated that blood NfL concentration is not influenced by blood-brain barrier permeability (Gisslén et al. 2015; Kalm et al. 2017).

Our primary aim was to assess the correlation between CSF and plasma NfL in PWH on virally suppressive ART and in lifestyle-similar HIV-negative individuals. Secondly, we aimed to determine factors associated with CSF and plasma NfL in PWH on virally suppressive ART and lifestyle-similar HIV-negative individuals.

\section{Methods}

\section{Participants}

The 'COmorBidity in Relation to AIDS' (COBRA) cohort was a study of PWH aged $\geq 45$ years, with viral suppression on ART for $\geq 12$ months, recruited between January 2013 and October 2014 at two large HIV treatment centres in Amsterdam, The Netherlands and London, UK (De Francesco et al. 2018). Age- and lifestyle-similar HIVnegative individuals were recruited at sexual health clinics and community groups.

For this sub-study, a stored plasma sample from each participant's baseline visit was retrieved. Participant demographics, medical history, lifestyle details and biological samples (CSF and blood) were obtained within the context of the study protocol and all participants gave written informed consent for future use of their stored data and samples.

Additionally, blood-based materials from 35 age-matched unselected blood bank donors were obtained from the Dutch national blood bank in Amsterdam, The Netherlands (www. sanquin.nl), as part of a separate sub-study within the main COBRA study (Booiman et al. 2017). Age and gender information were available for the blood bank donors. Blood bank donors from The Netherlands are routinely screened for blood-borne infections such as HIV, HTLV, hepatitis B, hepatitis $\mathrm{C}$ and syphilis. Individuals over the age of 70 years or those with high-risk behaviour for acquiring blood borne infections are excluded from blood donation. Overall, blood bank donors are at lower risk of acquiring blood-borne infections than the general population, but are often used as a control group for comparative studies.

I retrieved stored plasma samples from the blood bank donors who were included in the previous COBRA substudy, and measured plasma NfL using the same technique as described below.

\section{NfL measurement}

CSF NfL was measured previously in the COBRA study, using sandwich ELISA (NF-light ELISA kit; UmanDiagnostics AB, Umeå, Sweden) (De Francesco et al. 2018). For this sub-study, plasma NfL was measured using a NF-light digital immunoassay on the Simoa HD-1 platform (Quanterix Corp., Boston, MA, USA), at the UK Dementia Research Institute, University College London, UK (Hendricks et al. 2019). The lower limit of quantification (LLOQ) determined by the manufacturer is $0.174 \mathrm{pg} / \mathrm{mL}$. Samples were analysed in duplicate, diluted 1:4 and results were all above the LLOQ. Analyses were performed by the same technician using a single batch of reagents. The intra- and interassay coefficients of variations were below $6 \%$ and $15 \%$, respectively.

\section{Other laboratory measurements}

Laboratory parameters measured previously in the COBRA study included blood $\left(\mathrm{CD} 4^{+}\right.$and $\mathrm{CD} 8^{+}$counts, neopterin, albumin, creatinine and HIV RNA) and CSF (neopterin, albumin and HIV RNA). Blood and CSF HIV RNA were measured using the Abbott RealTime M2000 assay (Abbot, Chicago, USA) with a lower limit of detection of 40 copies $/ \mathrm{mL}$. Estimated glomerular filtration rate was calculated using the Chronic Kidney Disease Epidemiology Collaboration (CKD-EPI) formula (Levey et al. 2009).

\section{Statistical analyses}

Statistical analyses were performed using SAS version 9.4 (SAS Institute, Cary, NC, USA). P values $<0.05$ were considered statistically significant throughout. Differences in CSF and plasma NfL concentrations between PWH, HIV-negative participants and blood bank donors (where applicable) were tested for significance using Wilcoxon's test. The correlation between CSF and plasma NfL (after log10-transformation) was assessed using Pearson's correlation coefficient and the difference between groups was tested for significance using the Z-test after Fisher's transformation. $\log _{10}$-transformed $\mathrm{CSF}$ and plasma NfL Z-scores were calculated to enable direct comparisons between CSF and plasma NfL. A Bland-Altman graph (Bland and Altman 1999) was plotted to describe the agreement between $\log _{10}$-transformed CSF and plasma NfL 
Z-scores. This represents a plot of the difference between the CSF and plasma NfL Z-score against their average for each individual. Linear regression models were used to identify factors independently associated with $\log _{10}$-transformed CSF and plasma NfL, standardised to Z-scores with a mean of 0 and standard deviation of 1, separately in PWH and HIV-negative participants. Factors associated with either CSF or plasma NfL in either PWH or HIV-negative participants in univariable analyses, were included in multivariable models. Factors included in the univariable linear regression analysis were female gender, age, Black African ethnicity, men who have sex with men sexual orientation, weight, on hypertensive medication, body mass index, current tobacco smoker, ex-tobacco smoker, current alcohol consumption, past alcohol consumption, recent recreational drug use, ever used injected recreational drugs, global cognitive function T-score, current $\mathrm{CD}^{+}$and $\mathrm{CD} 8^{+}$ T cell counts, current $\mathrm{CD}^{+} / 8^{+}$ratio, serum creatinine, serum albumin, cerebrospinal fluid protein, cerebrospinal fluid neopterin and plasma neopterin. HIV-specific parameters significantly associated in univariable analyses were added to an additional model fitted in PWH only. The HIV-specific factors included in the univariable linear regression analysis included time since HIV diagnosis, time on ART, nadir CD4 ${ }^{+}$count, years with $\mathrm{CD}^{+}{ }^{+}$count $<200$ cells/ $\mu \mathrm{L}$, prior AIDS diagnosis, on a non-nucleoside reverse transcriptase inhibitor (NNRTI), on a protease inhibitor (PI), on tenofovir-disoproxil fumarate (TDF) and on atazanavir.

\section{Results}

Of the COBRA participants, 132/134 PWH and 79/79 HIVnegative participants and all 35 blood bank donors who were included in the previous COBRA sub-study had sufficient stored plasma available and were included in this analysis. Socio-demographic, lifestyle characteristics and anthropometrics at study baseline were described previously (De Francesco et al. 2018). Briefly, PWH and HIVnegative participants in this sub-study were similar in terms of age, gender, sexual orientation, anthropometric measurements, years of education and lifestyle factors. PWH were less likely to be of white ethnicity $(p=0.02)$ and current alcohol drinkers $(\mathrm{p}=0.01)$ than HIV-negative participants (Table 1).

Blood bank donors were similar in age (median (IQR) 59 years $(52,65))$ to the COBRA participants $(\mathrm{p}=0.41)$, but a higher proportion of blood bank donors were female $(48.6 \%)$, compared to the COBRA cohort $(\mathrm{p}<0.001)$.

PWH had been diagnosed for a median (IQR) of 15.0 $(9.0,19.6)$ years with a median (IQR) current $\mathrm{CD}^{+}{ }^{+}$count of $618(472,809)$ cells $/ \mu \mathrm{L}$, current CD4:CD8 ratio of 0.83 $(0.60,1.11)$ and a nadir CD4 ${ }^{+}$count of $175(85,240)$ cells/ $\mu \mathrm{L}$. PWH had been on ART for a median (IQR) of 12.8 (7.8,
16.9) years, all PWH had undetectable plasma HIV RNA and 130/132 had undetectable CSF HIV RNA (Table 1).

Median (IQR) CSF:serum albumin ratio (a marker of blood-brain barrier dysfunction) was similar between the groups $(6.9(4.9,8.5)$ and $6.2(5.0,7.5)$ in PWH and HIVnegative participants, respectively). Serum creatinine was significantly higher in PWH, median (IQR) $85.5(76,100)$ vs $79(72,92) \mu \mathrm{mol} / \mathrm{L}$ in PWH and HIV-negative participants respectively, $\mathrm{p}=0.03$ (Table 1 ).

\section{CSF and plasma NfL results}

Comparing PWH and HIV-negative participants, neither CSF NfL (median (IQR) $570(430,864)$ vs 568 (408, 767) $\mathrm{pg} / \mathrm{mL}, \mathrm{p}=0.37$ ), nor plasma NfL (median (IQR) 10.7 (7.9, 14.9) vs $9.9(7.2,12.5) \mathrm{pg} / \mathrm{mL}, \mathrm{p}=0.15)$ differed significantly between the groups. The correlation between CSF and plasma NfL did not differ significantly by HIV status (PWH: $r=0.52$ (95\% confidence interval (CI) 0.38-0.63); HIV-negative participants: $r=0.47$ (95\%CI 0.27-0.62), $p$ value testing the difference $=0.63)($ Fig. 1).

Plasma NfL was significantly lower in the blood bank donors cohort (median (IQR) 6.15 (4.94, 7.15)) compared to people with HIV $(\mathrm{p}<0.001)$ and HIV-negative participants $(\mathrm{p}<0.001)$.

Bland-Altman plots are reported in Fig. 2. Among PWH, there was not a systematic over- or under-estimation of one score compared to the other. Mean $(95 \% \mathrm{CI})$ difference between the scores was $0.00(-0.17,0.17), \mathrm{p}=0.99$ and the agreement between the two scores was not altered depending on the average CSF and plasma NfL Z-score; the correlation (95\% CI) between the difference and the mean of the two scores was $-0.004(-0.18,0.17), p=0.96$. The lower and upper limits of agreement $(95 \% \mathrm{CI})$ between the two measurements were $-1.97(-2.25,-1.68)$ and $1.97(1.68,2.25)$, respectively, indicating substantial discrepancies between the NfL Z-scores obtained from the two compartments. A similar pattern was observed among HIV-negative individuals, with a mean $(95 \% \mathrm{CI})$ difference of $0.00(-0.23,0.23)$ and no correlation between the difference and average $[\mathrm{r}$ $(95 \% \mathrm{CI})=0.00(-0.22,0.22), \mathrm{p}=0.99]$.

\section{Factors associated with CSF and plasma NfL}

In multivariable regression analysis, higher CSF NfL $\mathrm{Z}$-score was associated with older age and higher CSF protein in both PWH and HIV-negative participants; lower CSF NfL Z-score was significantly associated with female gender in HIV-negative participants $\left(0.83 \log _{10}\right.$ CSF NfL $\mathrm{Z}$-score lower compared to men, $\mathrm{p}=0.03$ ) and higher $\mathrm{CSF}$ NfL Z-score approached a statistically significant association with lower bodyweight in PWH (0.05 $\log _{10}$ CSF NfL $\mathrm{Z}$-score higher per $5 \mathrm{~kg}$ decrease in bodyweight, $\mathrm{p}=0.05$ ) 
Table 1 Sociodemographic, anthropometric, lifestyle and HIV-related characteristics of study participants

\begin{tabular}{|c|c|c|c|}
\hline Variable, $n(\%)$ or median (IQR) & $\begin{array}{l}\text { PWH } \\
(N=132)\end{array}$ & $\begin{array}{l}\text { HIV-negative } \\
\text { participants }(N=79)\end{array}$ & $p$ value \\
\hline Gender & & & 0.66 \\
\hline Female & $8(6.1 \%)$ & $6(7.6 \%)$ & \\
\hline Male & $124(93.9 \%)$ & $73(92.4 \%)$ & \\
\hline Ethnicity & & & 0.02 \\
\hline Black-African & $16(12.2 \%)$ & $2(2.6 \%)$ & \\
\hline White & $115(87.8 \%)$ & $76(97.4 \%)$ & \\
\hline Sexual orientation & & & 0.39 \\
\hline Men who have sex with men & $103(78.0 \%)$ & $59(74.7 \%)$ & \\
\hline Bisexual & $10(7.6 \%)$ & $4(5.1 \%)$ & \\
\hline Heterosexual & $18(13.6 \%)$ & $16(20.2 \%)$ & \\
\hline Age, years & $56(51,62)$ & $57(52,64)$ & 0.30 \\
\hline Years of education & $14(13,16)$ & $16(14,17)$ & 0.21 \\
\hline Weight, kg & $79.6(70.0,87.7)$ & $81.2(72.5,91.0)$ & 0.14 \\
\hline BMI, $\mathrm{kg} / \mathrm{m}^{2}$ & $24.7(22.7,27.5)$ & $24.6(23.2,28.4)$ & 0.35 \\
\hline Current smoker & $39(29.6 \%)$ & $20(25.3 \%)$ & 0.22 \\
\hline Years of smoking (current/ex-smokers) & $29(19,37)$ & $26(15,39)$ & 0.40 \\
\hline Current alcohol drinker & $103(78.0 \%)$ & $71(91.0 \%)$ & 0.04 \\
\hline Years of drinking (current/ex drinkers) & $29(19,37)$ & $41(35,47)$ & 0.01 \\
\hline Alcohol units/week (current drinkers) & $5.5(1.5,15.0)$ & $7.5(1.5,17.5)$ & 0.39 \\
\hline Ever used injected drugs & $5(3.8 \%)$ & $0(0.0 \%)$ & 0.16 \\
\hline Use of recreational drugs in past 6 months & $43(32.6 \%)$ & $18(22.8 \%)$ & 0.13 \\
\hline On hypertensive medication & $29(22.0 \%)$ & $14(17.7 \%)$ & 0.46 \\
\hline CSF albumin, mg/L & $299(210,368)$ & $267(214,326)$ & 0.43 \\
\hline Serum albumin, $g / L$ & $43(41,46)$ & $43(40,45)$ & 0.08 \\
\hline CSF:serum albumin & $6.9(4.9,8.5)$ & $6.2(5.0,7.5)$ & 0.38 \\
\hline Creatinine, $\mathrm{mmol} / \mathrm{L}$ & $85.5(76,100)$ & $79(72,92)$ & \\
\hline eGFR, $\mathrm{mL} / \mathrm{min}$ per $1.73 \mathrm{~m}^{2}$ & $85.5(71.0,97.3)$ & $92.0(77.4,102.3)$ & 0.03 \\
\hline Years since HIV diagnosis & $15.0(9.0,19.6)$ & N/A & N/A \\
\hline Duration of ART, years & $12.8(7.8,16.9)$ & N/A & N/A \\
\hline CSF HIV RNA viral load $<40$ copies $/ \mathrm{mL}$ & $130(98.5 \%)$ & N/A & N/A \\
\hline $\mathrm{CD}^{+} / \mathrm{CD}^{+}{ }^{+} \mathrm{T}$ cell count ratio & $0.83(0.60,1.11)$ & $2.00(1.44,2.65)$ & N/A \\
\hline Prior AIDS event & $42(31.8 \%)$ & N/A & N/A \\
\hline $\mathrm{CD}^{+} \mathrm{T}$ cell count $[$ cells $/ \mu \mathrm{L}]$ & $618(472,809)$ & $900(692,1174)$ & N/A \\
\hline Nadir $\mathrm{CD} 4^{+} \mathrm{T}$ cell count $[$ cells $/ \mu \mathrm{L}]$ & $175(85,240)$ & N/A & N/A \\
\hline Months with $\mathrm{CD}^{+} \mathrm{T}$ cell count $<200$ cells $/ \mu \mathrm{L}$ & $1.6(0.0,9.8)$ & N/A & N/A \\
\hline
\end{tabular}

$P W H$ people with HIV, BMI body mass index, $C S F$ cerebrospinal fluid, $e G F R$ estimated glomerular filtration rate, $A R T$ antiretroviral treament
(Table 2). Higher plasma NfL Z-score was associated with older age, higher serum creatinine and lower bodyweight in both PWH and HIV-negative participants, and higher CSF NfL Z-score approached a statistically significant association with lower serum albumin in PWH $\left(0.31 \log _{10} \mathrm{CSF}\right.$ $\mathrm{NfL} Z$-score higher per $10 \mathrm{~g} / \mathrm{L}$ decrease in serum albumin, $\mathrm{p}=0.05)($ Table 2).

Among PWH, none of the HIV-specific parameters showed a significant association with either CSF and plasma $\mathrm{NfL} \mathrm{Z-scores,} \mathrm{after} \mathrm{accounting} \mathrm{for} \mathrm{other} \mathrm{factors} \mathrm{(Table} \mathrm{3).}$

\section{Discussion}

In this study, we observed that the correlation between CSF and plasma NfL in PWH is moderate and similar to that observed in a cohort of lifestyle-similar HIV-negative controls. The previous report suggesting a strong correlation in a cohort of mainly untreated PWH (Gisslén et al. 2015 ) is likely a consequence of the much higher concentrations of CSF and plasma NfL seen in that cohort, where the median CSF and plasma NfL concentrations in 


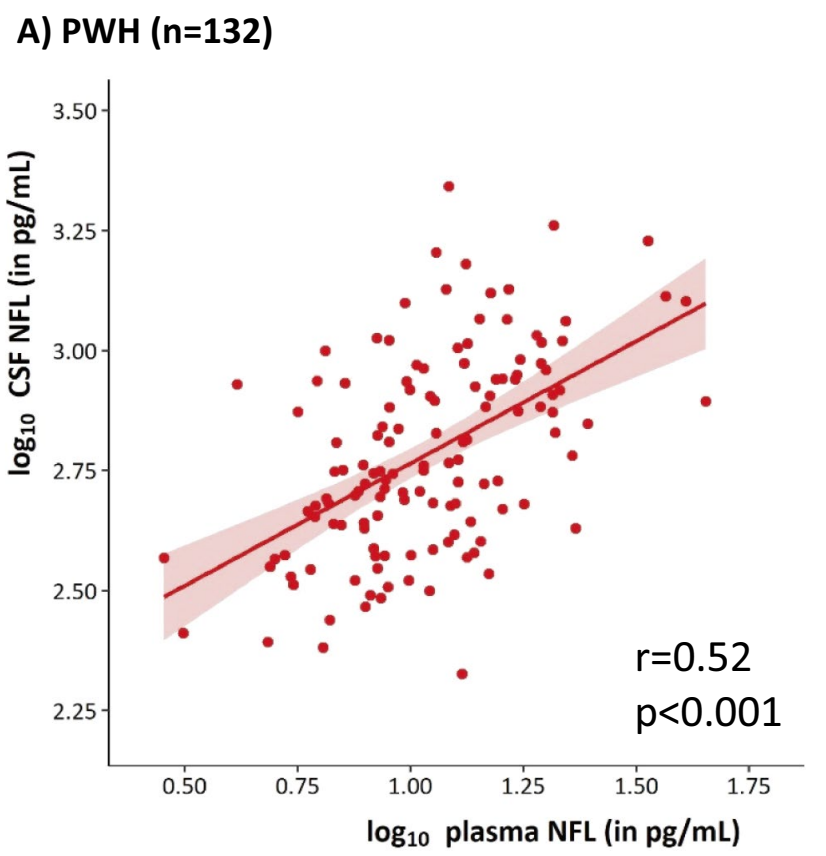

\section{B) HIV-negative $(n=79)$}

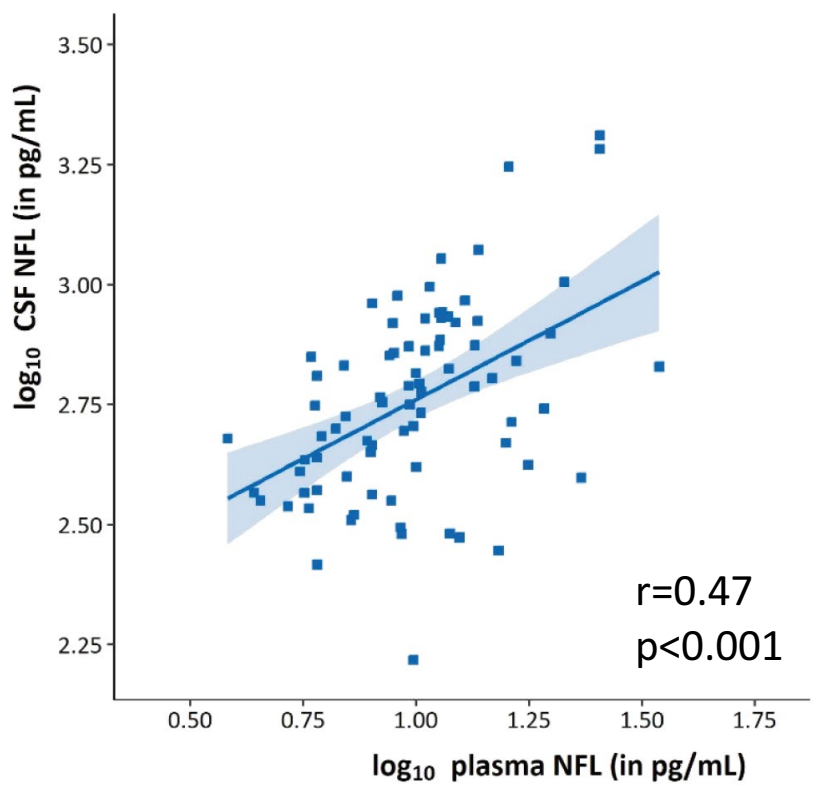

Fig. 1 Correlation between log-transformed plasma and CSF NFL concentration in PWH (A) and HIV-negative controls (B)

individuals with HIV-associated dementia were $16,185 \mathrm{pg} /$ $\mathrm{mL}$ and $114 \mathrm{pg} / \mathrm{mL}$, respectively.

Concentrations of CSF and plasma NfL were similar in PWH and HIV-negative participants, suggesting that PWH on ART do not have increased biomarker evidence of neuroaxonal injury compared to lifestyle-similar HIV-negative individuals. CSF/plasma NfL ratios also did not differ by
HIV status, with plasma NfL being 55-65 times lower compared to CSF NfL, consistent with published literature (Gisslén et al. 2015). In view of the lack of significant difference in cerebrospinal fluid Nfl, plasma NfL and cerebrospinal fluid/plasma NfL ratios between PWH on suppressive ART and lifestyle-similar HIV-negative participants, we did not correlate our findings with the cerebral imaging data
A) PWH $(n=132)$

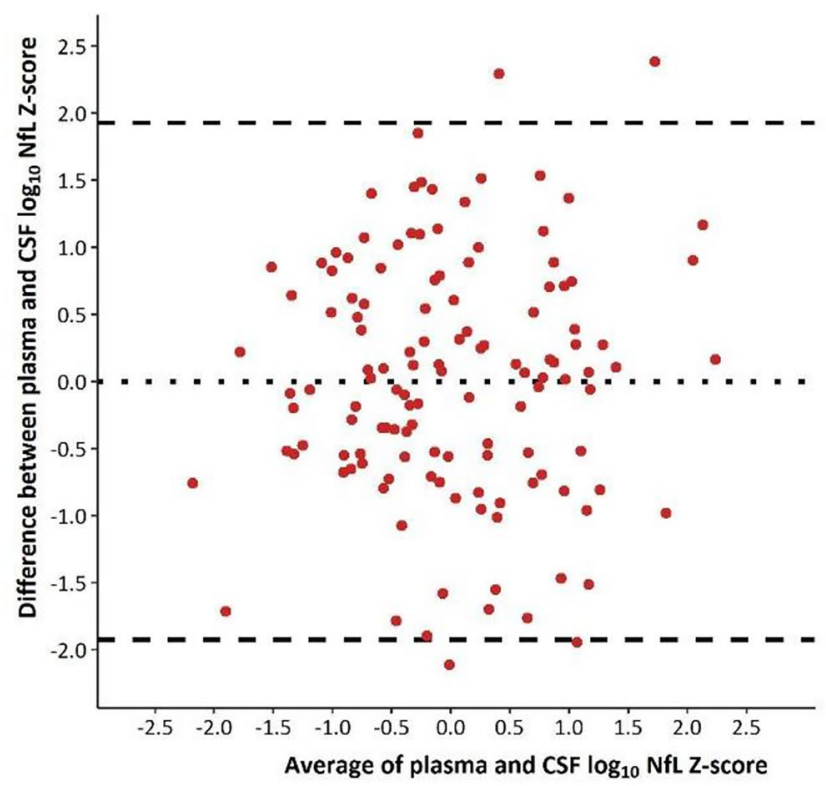

B) HIV-negative participants ( $n=79$ )

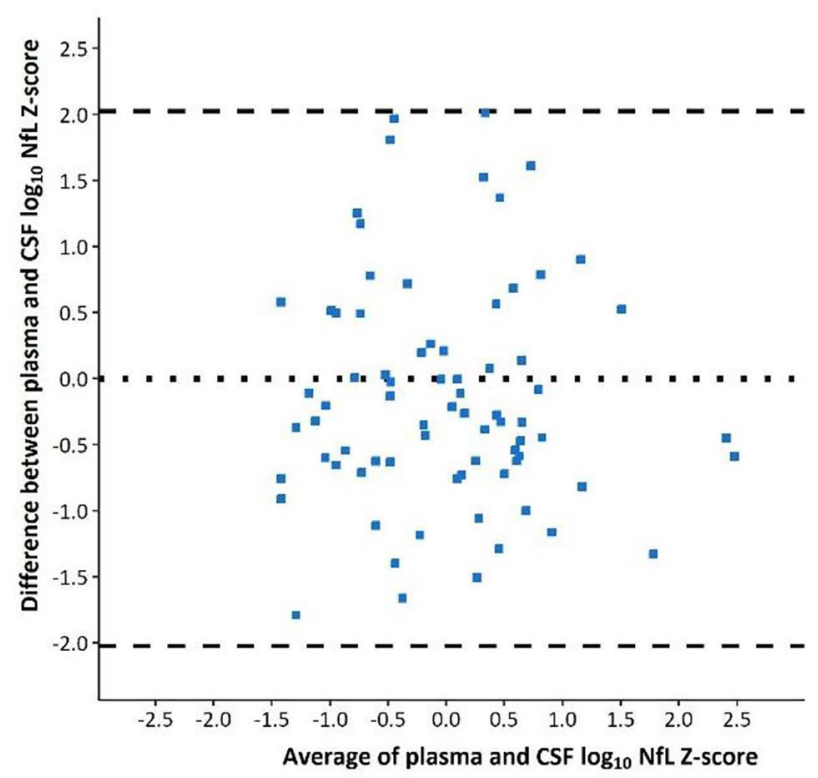

Fig. 2 Bland-Altman plot of plasma and CSF NFL Z-scores in PWH (A) and HIV-negative participants (B) 


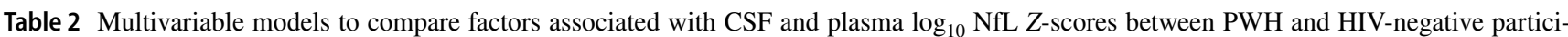
pants, excluding HIV-specific parameters ${ }^{\mathrm{a}, \mathrm{b}}$

\begin{tabular}{|c|c|c|c|c|c|}
\hline & \multirow[t]{2}{*}{ Variable } & \multicolumn{2}{|l|}{ PWH } & \multicolumn{2}{|l|}{ HIV-negative controls } \\
\hline & & Change (95\% CI) & $p$ value & Change (95\% CI) & $p$ value \\
\hline \multirow[t]{9}{*}{$\log _{10}$ CSF NFL $Z$-score } & Female vs male & $-0.44(-1.05,0.17)$ & 0.15 & $-0.83(-1.56,-0.10)$ & 0.03 \\
\hline & Age (per 10 years) & $0.69(0.50,0.88)$ & $<0.001$ & $0.74(0.46,1.02)$ & $<0.001$ \\
\hline & Weight (per 5 kg) & $-0.05(-0.10,0.00)$ & 0.05 & $0.02(-0.04,0.07)$ & 0.55 \\
\hline & Hypertensive medication (yes vs no) & $0.26(-0.08,0.61)$ & 0.13 & $-0.05(-0.64,0.55)$ & 0.87 \\
\hline & Serum creatinine (per mg/dL) & $0.47(-0.29,1.24)$ & 0.22 & $-0.71(-2.00,0.59)$ & 0.28 \\
\hline & Serum albumin (per $10 \mathrm{~g} / \mathrm{L})$ & $0.00(-0.33,0.34)$ & 0.98 & $0.09(-0.50,0.67)$ & 0.77 \\
\hline & CSF protein (per g/L) & $1.46(0.63,2.29)$ & $<0.001$ & $1.81(0.37,3.25)$ & 0.01 \\
\hline & CSF neopterin & $-0.01(-0.04,0.01)$ & 0.33 & $0.04(-0.06,0.13)$ & 0.45 \\
\hline & Plasma neopterin & $0.02(-0.01,0.05)$ & 0.18 & $-0.002(-0.11,0.11)$ & 0.96 \\
\hline \multirow[t]{9}{*}{$\log _{10}$ plasma NFL $Z$-score } & Female vs male & $0.13(-0.44,0.69)$ & 0.66 & $0.57(-0.13,1.27)$ & 0.11 \\
\hline & Age (per 10 years) & $0.62(0.44,0.80)$ & $<0.001$ & $0.63(0.36,0.90)$ & $<0.001$ \\
\hline & Weight (per 5 kg) & $-0.13(-0.18,-0.08)$ & $<0.001$ & $-0.06(-0.11,0.00)$ & 0.05 \\
\hline & Hypertensive medication (yes vs no) & $0.28(-0.03,0.60)$ & 0.08 & $0.18(-0.39,0.76)$ & 0.53 \\
\hline & Serum creatinine (per mg/dL) & $0.73(0.02,1.44)$ & 0.04 & $1.64(0.39,2.89)$ & 0.01 \\
\hline & Serum albumin (per $10 \mathrm{~g} / \mathrm{L})$ & $-0.31(-0.63,0.00)$ & 0.05 & $-0.39(-0.95,0.18)$ & 0.18 \\
\hline & CSF protein (per g/L) & $-0.30(-1.07,0.47)$ & 0.44 & $0.45(-0.94,1.84)$ & 0.52 \\
\hline & CSF neopterin & $0.01(-0.02,0.03)$ & 0.69 & $0.08(-0.01,0.17)$ & 0.07 \\
\hline & Plasma neopterin & $0.03(0.00,0.05)$ & 0.07 & $0.01(-0.09,0.11)$ & 0.85 \\
\hline
\end{tabular}

${ }^{\text {a }}$ Parameter estimates reflect the associated impact (measured in standard deviations) of each independent variable in the model on the dependent variable

${ }^{\mathrm{b}}$ Variables included in the univariable linear regression analysis that were not statistically significant and not included in the multivariable model were Black African ethnicity, men who have sex with men sexual orientation, body mass index, current tobacco smoker, ex-tobacco smoker, current alcohol consumption, past alcohol consumption, recent recreational drug use, ever used injected recreational drugs, global cognitive function $T$-score, current $\mathrm{CD} 4^{+}$and $\mathrm{CD} 8^{+}$count and current $\mathrm{CD} 4^{+} / 8^{+}$ratio

from this cohort, given that these data have already been previously reported.

Our results demonstrated that plasma NfL concentration was significantly lower in the blood bank donors compared to the PWH and HIV-negative controls enrolled into the COBRA study. Given the lack of additional demographic factors apart from age and gender, and concurrent cerebrospinal fluid samples, we were unable to characterise this further, and can only conclude that based on the limited information available, PWH and HIV-negative controls in the COBRA study were observed to have evidence of higher plasma NfL concentrations as a biomarker of neuro-axonal injury, compared to blood bank donors.

Our results demonstrating that higher CSF and plasma $\mathrm{NfL}$ were associated with older age echoes the findings in published studies (Disanto et al. 2017; Yilmaz et al. 2017). The finding that higher CSF NfL is associated with higher CSF protein is unsurprising, given that higher CSF protein may indicate CNS pathology, and hence higher neuronal damage. Our finding that female gender is associated with lower cerebrospinal fluid NfL is not wholly unexpected, though the published data in this regard remains controversial with some studies demonstrating that cerebrospinal fluid
NfL is higher in males (Forgrave et al. 2019; Lin et al. 2018), higher in females (Lu et al. 2015) or not significantly different by gender (Manouchehrinia et al. 2020a, b).

Our results suggest that consideration of renal function and body composition is an important requirement when interpreting plasma NfL. While renal excretion of NfL has not been extensively described, NfL has a molecular weight of about $68 \mathrm{kDa}$ and a high abundance of negatively charged amino acids in its sequence, both of which theoretically decrease its ability to cross the glomerular filtration barrier. Published data demonstrate that while plasma NfL decreased after switching from tenofovir disoproxil fumarate to tenofovir alafenamide fumarate, no significant correlations between plasma NfL and serum creatinine pre- and post-switch, or between changes in plasma $\mathrm{NfL}$ and serum creatinine pre- and post-switch were observed (Hermansson et al. 2019). Although lower bodyweight was independently associated with higher plasma NfL, body mass index was not an associated factor in our study, in contrast to a publication demonstrating a negative association between plasma NfL and body mass index (Manouchehrinia et al. 2020a, b).

Strengths of our study include the comprehensivelyphenotyped cohort and the lifestyle-similar HIV-negative 
Table 3 Multivariable models to assess factors associated with CSF and plasma $\log _{10}$ NFL Z-scores in PWH only, and including HIV-related parameters $^{\mathrm{a}, \mathrm{b}}$

\begin{tabular}{|c|c|c|c|}
\hline & \multirow[t]{2}{*}{ Variable } & \multicolumn{2}{|l|}{ PWH } \\
\hline & & Change (95\% CI) & $p$ value \\
\hline \multirow{11}{*}{$\log _{10}$ CSF NFL $Z$-score } & Female vs male & $-0.48(-1.09,0.14)$ & 0.13 \\
\hline & Age (per 10 years) & $0.69(0.49,0.89)$ & $<0.001$ \\
\hline & Weight (per 5 kg) & $-0.06(-0.11,-0.01)$ & 0.03 \\
\hline & Hypertensive medication (yes vs no) & $0.28(-0.06,0.63)$ & 0.11 \\
\hline & Serum creatinine (per mg/dL) & $0.41(-0.36,1.18)$ & 0.30 \\
\hline & Serum albumin (per $10 \mathrm{~g} / \mathrm{L})$ & $-0.01(-0.35,0.33)$ & 0.96 \\
\hline & CSF protein (per g/L) & $1.37(0.53,2.21)$ & 0.002 \\
\hline & CSF neopterin & $-0.01(-0.04,0.01)$ & 0.34 \\
\hline & Plasma neopterin & $0.02(-0.01,0.05)$ & 0.22 \\
\hline & Time since HIV diagnosis (years) & $0.18(-0.22,0.59)$ & 0.38 \\
\hline & Time on cART (years) & $-0.35(-0.81,0.11)$ & 0.14 \\
\hline \multirow[t]{11}{*}{$\log _{10}$ plasma NFL $Z$-score } & Female vs male & $0.18(-0.38,0.75)$ & 0.52 \\
\hline & Age (per 10 years) & $0.58(0.39,0.76)$ & $<0.001$ \\
\hline & Weight (per 5 kg) & $-0.12(-0.17,-0.07)$ & $<0.001$ \\
\hline & Hypertensive medication (yes vs no) & $0.25(-0.07,0.57)$ & 0.12 \\
\hline & Serum creatinine (per mg/dL) & $0.79(0.08,1.50)$ & 0.03 \\
\hline & Serum albumin (per $10 \mathrm{~g} / \mathrm{L}$ ) & $-0.29(-0.60,0.02)$ & 0.07 \\
\hline & CSF protein (per g/L) & $-0.23(-1.00,0.55)$ & 0.56 \\
\hline & CSF neopterin & $0.01(-0.02,0.03)$ & 0.53 \\
\hline & Plasma neopterin & $0.03(0.00,0.06)$ & 0.05 \\
\hline & Time since HIV diagnosis (years) & $0.30(-0.08,0.67)$ & 0.12 \\
\hline & Time on cART (years) & $-0.17(-0.59,0.26)$ & 0.44 \\
\hline
\end{tabular}

a Parameter estimates reflect the associated impact (measured in standard deviations) of each independent variable in the model on the dependent variable

${ }^{\mathrm{b}}$ Variables included in the univariable linear regression analysis that were not statistically significant and not included in the multivariable model were Black African ethnicity, men who have sex with men sexual orientation, body mass index, current tobacco smoker, ex-tobacco smoker, current alcohol consumption, past alcohol consumption, recent recreational drug use, ever used injected recreational drugs, global cognitive function $T$-score, current $\mathrm{CD} 4^{+}$and $\mathrm{CD} 8^{+}$count, current $\mathrm{CD} 4^{+} / 8^{+}$ratio, nadir $\mathrm{CD} 4^{+}$count, years with $\mathrm{CD}^{+}{ }^{+}$count $<200$ cells/ $\mu \mathrm{L}$, prior AIDS diagnosis, on a non-nucleoside reverse transcriptase inhibitor (NNRTI), on a protease inhibitor (PI), on tenofovir-disoproxil fumarate (TDF) and on atazanavir individuals. Limitations include the COBRA participants being predominantly middle-aged white men who have sex with men from Western Europe, thus more data on the correlation between CSF and plasma NFL are required from other populations, as it is unknown whether our findings can be extrapolated to other populations. Further work will need to be performed to assess plasma NfL in younger PWH who may not have been on suppressive ART for as long as the COBRA study participants. Another limitation was the lack of data on other factors that may increase plasma NFL (e.g. peripheral neuropathy) and concomitant neurotoxic drug use. The number of female participants in our study was small and studies involving more female participants would be needed to verify the association between female gender and lower CSF NfL.

Our results demonstrate that cerebrospinal fluid and plasma NfL concentrations are similar in people with
HIV on suppressive antiretroviral treatment and in lifestyle-similar HIV-negative individuals. Furthermore, our findings suggest that plasma NfL may be a promising and more easily accessible surrogate biomarker of neuronal integrity. Cerebrospinal fluid NfL measurements closely reflect processes taking place within the CNS, whereas both the CNS and PNS contribute to plasma NfL measurements, which may lead to wider variations in plasma NfL measurements. In view of this, further work is required to improve our understanding of the factors that influence plasma NfL concentrations.

Acknowledgements We would like to thank the following groups and individuals for their contributions:

The Writing Group: Davide De Francesco ${ }^{1 *}$, Ferdinand W. Wit ${ }^{2,12}$, James H. Cole ${ }^{3}$, Neeltje A. Kootstra ${ }^{2}$, Alan Winston ${ }^{3}$, Caroline A. Sabin ${ }^{1}$ Jonathan Underwood ${ }^{3}$, Rosan A. van Zoest ${ }^{2}$, Judith Schouten ${ }^{2}$, Katherine W. Kooij ${ }^{2}$, Maria Prins ${ }^{4}$, Giovanni Guaraldi ${ }^{5}$, Matthan W.A. Caan ${ }^{2}$, David 
Burger ${ }^{6}$, Claudio Franceschi ${ }^{7}$, Claude Libert ${ }^{8}$, Alexander Bürkle ${ }^{9}$ and Peter Reiss ${ }^{2}$. The COBRA Steering Committee: P. Reiss ${ }^{2}$ (chair), A. Winston ${ }^{3}$, F.W. Wit ${ }^{2,12}$, M. Prins ${ }^{4}$, M.F. Schim van der Loeff ${ }^{4}$, J. Schouten ${ }^{2}$, B. Schmand $^{2}$, G.J. Geurtsen ${ }^{2}$, D.J. Sharp ${ }^{3}$, M.W.A. Caan ${ }^{2}$, C. Majoie ${ }^{2}$, J. Villaudy $^{2}$, B. Berkhout ${ }^{2}$, N.A. Kootstra ${ }^{2}$, M. Gisslén ${ }^{10}$, A. Pasternak ${ }^{2}$, C.A. Sabin ${ }^{1}$, G. Guaraldi ${ }^{5}$, A. Bürkle ${ }^{9}$, C. Libert $^{8}$, C. Franceschi ${ }^{7}$, A. Kalsbeek $^{2}$, E. Fliers ${ }^{2}$, J. Hoeijmakers ${ }^{11}$, J. Pothof ${ }^{11}$, M. van der Valk ${ }^{2}$, P.H. Bisschop $^{2}$, P. Portegies ${ }^{2}$, S. Zaheri ${ }^{12}$ and D. Burger ${ }^{6}$. The COBRA Project Management Board: P. Reiss ${ }^{2}$, A. Winston ${ }^{3}$, F.W. Wit ${ }^{2,12}$, J.H. Cole ${ }^{3}$, M.W.A. Caan ${ }^{2}$, J. Villaudy ${ }^{2}$, N.A. Kootstra ${ }^{2}$, M.F. Schim van der Loeff ${ }^{4}$, M. Gisslén ${ }^{10}$, C.A. Sabin ${ }^{1}$, A. Bürkle ${ }^{9}$ and W. Zikkenheiner ${ }^{2}$. The Management Team: P. Reiss ${ }^{2}$, W. Zikkenheiner ${ }^{2}$, F.W. Wit ${ }^{2,12}$, F.R. Janssen ${ }^{2}$. The Clinical Cohort Team: A. Winston ${ }^{3}$, F.W. Wit ${ }^{2,12}$, J. Underwood ${ }^{3}$, J. Schouten $^{2}$, K.W. Kooij ${ }^{2}$, R.A. van Zoest ${ }^{2}$, N. Doyle ${ }^{3}$, M. Prins ${ }^{4}$, M. Schim van der Loeff ${ }^{4}$, P. Portegies ${ }^{2}$, B.A. Schmand ${ }^{2}$, G.J. Geurtsen ${ }^{2}$, E. Verheij ${ }^{2}$, S.O. Verboeket ${ }^{2}$, B.C. Elsenga ${ }^{2}$, M. van der Valk ${ }^{2}$, S. Zaheri ${ }^{12}$, M.M.J. Hillebregt $^{12}$, Y.M.C. Ruijs ${ }^{12}$, D.P. Benschop ${ }^{12}$, L. Tembo ${ }^{3}$, L. McDonald ${ }^{3}$, M. Stott ${ }^{3}$, K. Legg ${ }^{3}$, A. Lovell ${ }^{3}$, O. Erlwein ${ }^{3}$, C. Kingsley ${ }^{3}$, P. Norsworthy ${ }^{3}$, S. Mullaney ${ }^{3}$, T. Kruijer ${ }^{2}$, L. del Grande ${ }^{2}$, V. Olthof ${ }^{2}$, G.R. Visser ${ }^{4}$, L. May $^{4}$, F. Verbraak ${ }^{2}$, N. Demirkaya ${ }^{2}$, I. Visser ${ }^{2}$, G. Guaraldi ${ }^{5}$. The Neuroimaging Team: D.J. Sharp ${ }^{3}$, M.W.A. Caan ${ }^{2}$, J.H. Cole ${ }^{3}$, C.B.L.M. Majoie ${ }^{2}$, T. $\mathrm{Su}^{2}, \mathrm{R}$. Leech $^{3}$, J. Huguet ${ }^{2}$. The HIS Mouse Study Team: J. Villaudy ${ }^{2}$, E. Frankin ${ }^{2}$, B. Berkhout ${ }^{2}$, A. van der Kuyl ${ }^{2}$, K. Weijer ${ }^{2}$, E. Siteur-Van Rijnstra $^{2}$, D. Burger ${ }^{6}$, M. de Graaff-Teulen ${ }^{6}$. The Biomarker Team: N.A. Kootstra $^{2}$, M. Gisslén ${ }^{10}$, A.M. Harskamp-Holwerda ${ }^{2}$, I. Maurer ${ }^{2}$, M.M. Mangas Ruiz ${ }^{2}$, A.F. Girigorie ${ }^{2}$, B. Boeser-Nunnink ${ }^{2}$, T. Booiman ${ }^{2}$, A. Kalsbeek $^{2}$, P.H.L.T. Bisschop ${ }^{2}$, D. Burger ${ }^{6}$, M. de Graaff-Teulen ${ }^{6}$, J. Hoeijmakers ${ }^{11}$, J. Pothof ${ }^{11}$, C. Libert ${ }^{8}$, S. Dewaele ${ }^{8}$, A. Pasternak ${ }^{2}$, C. Franceschi $^{7}$, P. Garagnani ${ }^{7}$, C. Pirazzini ${ }^{7}$, M. Capri ${ }^{7}$, F. Dall'Olio ${ }^{7}$, M. Chiricolo $^{7}$, S. Salvioli ${ }^{7}$, D. Fuchs ${ }^{10}$, H. Zetterberg ${ }^{10}$, D. Weber ${ }^{13}$, T. Grune $^{13}$, E.H.J.M. Jansen ${ }^{14}$. The Data Management and Analysis Team: C.A. Sabin ${ }^{1}$, D. De Francesco ${ }^{1}$, F.W. Wit ${ }^{2,12}$. The Dissemination Team: A. Bürkle $^{9}$, T. Sindlinger ${ }^{9}$, S. Oehlke ${ }^{9}$, W. Zikkenheiner ${ }^{2}$, R.A. van Zoest ${ }^{2}$.

Imperial College National Institute of Health Research (NIHR)

Biomedical Research Centre (BRC)

Sub-study project administration: $\mathrm{H}$. Box ${ }^{3}$

${ }^{1}$ University College London, London, UK.

${ }^{2}$ Academisch Medisch Centrum, Universiteit van Amsterdam, Amsterdam, The Netherlands.

${ }^{3}$ Imperial College London, London, UK.

${ }^{4}$ GGD Amsterdam, Public Health Service Amsterdam, Amsterdam,

The Netherlands

${ }^{5}$ Università degli studi di Modena e Reggio Emilia, Modena, Italy.

${ }^{6}$ Stichting Katholieke Universiteit Nijmegen, Nijmegen, The Netherlands.

${ }^{7}$ Alma Mater Studiorum Universita di Bologna, Bologna, Italy.

${ }^{8}$ Vlaams Instituut voor Biotechnologie, Ghent, Belgium.

${ }^{9}$ Universität Konstanz, Konstanz, Germany.

${ }^{10}$ Göteborgs Universitet, Göteborgs, Sweden.

${ }^{11}$ Erasmus Universitair Medisch Centrum Rotterdam, Rotterdam, The Netherlands.

${ }^{12}$ Stichting HIV Monitoring, Amsterdam, The Netherlands.

${ }^{13}$ German Institute of Human Nutrition, Potsdam, Germany.

${ }^{14}$ RIVM National Institute of Public Health and the Environment, Bilthoven, The Netherlands

Authors' contributions Jasmini Alagaratnam, Alan Winston and Sarah Fidler conceptualised the idea for the study and obtained the funding for the study. Neeltje Kootstra, Magnus Gisslén, Peter Reiss, Caroline A Sabin and Alan Winston established the COBRA consortium. Jamie Toombs undertook the laboratory work. Amanda Heslegrave and Henrik Zetterberg oversaw the laboratory work. Caroline A Sabin, Davide De Francesco, Jasmini Alagaratnam and Alan Winston undertook the statistical analyses of the results. Jasmini Alagaratnam wrote the first draft of the manuscript. All authors read and reviewed the final manuscript.
Funding This sub-study is independent research funded by grants awarded by the British HIV Association (BHIVA) and Imperial Health Charity.

The COBRA study was supported by a European Union's Seventh Framework Programme grant to the Comorbidity in Relation to AIDS (COBRA) project (FP-7-HEALTH 305,522), National Institute for Health Research (NIHR) Professorship (NIHR-RP-011-048), NIHR Imperial Biomedical Research Centre, the Netherlands Organisation for Health Research and Development (grant number 300020007) \& Stichting AIDS Fonds (grant number 2009063), Nuts-Ohra Foundation (grant number 1003-026) and unrestricted scientific grants from: Gilead Sciences, ViiV Healthcare, Janssen Pharmaceutica N.V. BristolMyers Squibb (BMS), and Merck \& Co to the AGEhIV cohort study, and investigator initiated grants from BMS, Gilead Sciences, Janssen, Merck and ViiV Healthcare to the POPPY cohort study.

A Wellcome Trust Multi-User Equipment grant to $\mathrm{HZ}$ and $\mathrm{AH}$ funded the instrument used for the biomarker measurements.

\section{Declarations}

Ethics approval This study was approved by the institutional review board of the Academic Medical Center (AMC) (reference number NL 30802.018.09) and a UK Research Ethics Committee (REC) (reference number 13/LO/0584 Stanmore, London).

Conflicts of interest JA has received financial support to attend scientific conferences from MSD, Janssen UK and Gilead Sciences. HZ has served on scientific advisory boards for Denali, Roche Diagnostics, Wave, Samumed and $\operatorname{CogRx}$; has given lectures in symposia sponsored by Fujirebio, Alzecure and Biogen; and is a co-founder of Brain Biomarker Solutions in Gothenburg AB, a GU Ventures-based platform company at the University of Gothenburg (outside submitted work). $\mathrm{JU}$ has received honoraria for preparation of educational materials and has served on an advisory board for Gilead Sciences. MG has received research grants from Gilead Sciences and Janssen-Cilag and honoraria as speaker and/or scientific advisor from Bristol-Myers Squibb, Gilead Sciences, GlaxoSmithKline/ViiV, Janssen-Cilag and MSD. PR through his institution has received independent scientific grant support from Gilead Sciences, Janssen Pharmaceuticals Inc., Merck \& Co and ViiV Healthcare, and has served on scientific advisory boards for Gilead Sciences, ViiV Healthcare, Merck \& Co. and Teva pharmaceutical industries, for which honoraria were all paid to his institution. AW has received honoraria or research grants on behalf of Imperial College London or been a consultant or investigator in clinical trials sponsored by Bristol-Myers Squibb, Gilead Sciences, GlaxoSmithKline, JanssenCilag, Roche and ViiV Healthcare. CAS has received funding for the membership of Data Safety and Monitoring Boards, Advisory Boards and for preparation of educational materials from Gilead Sciences and ViiV Healthcare. For the remaining authors, none were declared.

Open Access This article is licensed under a Creative Commons Attribution 4.0 International License, which permits use, sharing, adaptation, distribution and reproduction in any medium or format, as long as you give appropriate credit to the original author(s) and the source, provide a link to the Creative Commons licence, and indicate if changes were made. The images or other third party material in this article are included in the article's Creative Commons licence, unless indicated otherwise in a credit line to the material. If material is not included in the article's Creative Commons licence and your intended use is not permitted by statutory regulation or exceeds the permitted use, you will need to obtain permission directly from the copyright holder. To view a copy of this licence, visit http://creativecommons.org/licenses/by/4.0/. 


\section{References}

Abdulle S, Mellgren Å, Brew BJ, Cinque P, Hagberg L, Price RW, Rosengren L, Gisslén M (2007) CSF neurofilament protein (NFL) - A marker of active HIV-related neurodegeneration. J Neurol 254(8):1026-1032. https://doi.org/10.1007/s00415-006-0481-8

Bland JM, Altman DG (1999) Measuring agreement in method comparison studies. Stat Methods Med Res. https://doi.org/10.1177/ 096228029900800204

Booiman T, Wit FW, Girigorie AF, Maurer I, De Francesco D, Sabin CA, Harskamp AM, Prins M, Franceschi C, Deeks SG, Winston A, Reiss P, Kootstra NA (2017) Terminal differentiation of T cells is strongly associated with CMV infection and increased in HIV-positive individuals on ART and lifestyle matched controls. PLoS One. https://doi.org/10.1371/journal.pone.0183357

Bridel C, Van Wieringen WN, Zetterberg H, Tijms BM, Teunissen CE, Alvarez-Cermeño JC, Andreasson U, Axelsson M, Bäckström DC, Bartos A, Bjerke M, Blennow K, Boxer A, Brundin L, Burman J, Christensen T, Fialová L, Forsgren L, Frederiksen JL, Wild EJ (2019) Diagnostic Value of Cerebrospinal Fluid Neurofilament Light Protein in Neurology: A Systematic Review and Meta-analysis. JAMA Neurol. https://doi.org/10.1001/ jamaneurol.2019.1534

De Francesco D, Wit FW, Cole JH, Kootstra NA, Winston A, Sabin CA, Underwood J, Van Zoest RA, Schouten J, Kooij KW, Prins M, Guaraldi G, Caan MWA, Burger D, Franceschi C, Libert C, Bü Rkle A, Reiss P (2018) The "COmorBidity in Relation to AIDS" (COBRA) cohort: Design, methods and participant characteristics, on behalf of the COmorBidity in Relation to AIDS (COBRA) collaboration. PLoS One 13(3):e0191791. https://doi. org/10.1371/journal.pone.0191791

Disanto G, Barro C, Benkert P, Naegelin Y, Schädelin S, Giardiello A, Zecca C, Blennow K, Zetterberg H, Leppert D, Kappos L, Gobbi C, Kuhle J (2017) Serum Neurofilament light: A biomarker of neuronal damage in multiple sclerosis. Ann Neurol. https://doi.org/10.1002/ana.24954

Forgrave LM, Ma M, Best JR, DeMarco ML (2019) The diagnostic performance of neurofilament light chain in CSF and blood for Alzheimer's disease, frontotemporal dementia, and amyotrophic lateral sclerosis: a systematic review and meta-analysis. In Alzheimer's and Dementia: Diagnosis, Assessment and Disease Monitoring. https://doi.org/10.1016/j.dadm.2019.08.009

Gaetani L, Blennow K, Calabresi P, Di Filippo M, Parnetti L, Zetterberg $\mathrm{H}$ (2019) Neurofilament light chain as a biomarker in neurological disorders. In Journal of Neurology, Neurosurgery and Psychiatry. https://doi.org/10.1136/jnnp-2018-320106

Gisslén M, Hagberg L, Brew BJ, Cinque P, Price RW, Rosengren L (2007) Elevated Cerebrospinal Fluid Neurofilament Light Protein Concentrations Predict the Development of AIDS Dementia Complex. J Infect Dis 195(12):1774-1778. https://doi.org/10. 1086/518043

Gisslén M, Price RW, Andreasson U, Norgren N, Nilsson S, Hagberg L, Fuchs D, Spudich S, Blennow K, Zetterberg H (2015) Plasma concentration of the neurofilament light protein (NFL) is a biomarker of CNS injury in hiv infection: a cross-sectional study. EBioMedicine. https://doi.org/10.1016/j.ebiom.2015.11.036

Hansson O, Janelidze S, Hall S, Magdalinou N, Lees AJ, Andreasson U, Norgren N, Linder J, Forsgren L, Constantinescu R, Zetterberg H, Blennow K (2017) Blood-based NfL: a biomarker for differential diagnosis of parkinsonian disorder. Neurology. https://doi. org/10.1212/WNL.0000000000003680

Hendricks R, Baker D, Brumm J, Davancaze T, Harp C, Herman A, Von Büdingen HC, Townsend M, Fischer SK (2019) Establishment of neurofilament light chain Simoa assay in cerebrospinal fluid and blood. Bioanalysis. https://doi.org/10.4155/ bio-2019-0163
Hermansson L, Yilmaz A, Price RW, Nilsson S, McCallister S, Makadzange T, Das M, Zetterberg H, Blennow K, Gisslen M (2019) Plasma concentration of neurofilament light chain protein decreases after switching from tenofovir disoproxil fumarate to tenofovir alafenamide fumarate. PLoS One. https://doi.org/10. 1371/journal.pone.0226276

Kalm M, Boström M, Sandelius A, Eriksson Y, Ek CJ, Blennow K, Björk-Eriksson T, Zetterberg H (2017) Serum concentrations of the axonal injury marker neurofilament light protein are not influenced by blood-brain barrier permeability. Brain Res. https://doi. org/10.1016/j.brainres.2017.05.011

Khalil M, Teunissen CE, Otto M, Piehl F, Sormani MP, Gattringer T, Barro C, Kappos L, Comabella M, Fazekas F, Petzold A, Blennow K, Zetterberg H, Kuhle J (2018) Neurofilaments as biomarkers in neurological disorders. In Nature Reviews Neurology. https://doi. org/10.1038/s41582-018-0058-Z

Krut JJ, Mellberg T, Price RW, Hagberg L, Fuchs D, Rosengren L, Nilsson S, Zetterberg H, Gissl NM (2014) Biomarker evidence of axonal injury in neuroasymptomatic HIV-1 patients. PLoS One 9(2). https://doi.org/10.1371/journal.pone.0088591

Kuhle J, Barro C, Andreasson U, Derfuss T, Lindberg R, Sandelius A, Liman V, Norgren N, Blennow K, Zetterberg H (2016) Comparison of three analytical platforms for quantification of the neurofilament light chain in blood samples: ELISA, electrochemiluminescence immunoassay and Simoa. Clin Chem Lab Med. https:// doi.org/10.1515/cclm-2015-1195

Levey AS, Stevens LA, Schmid CH, Zhang Y, Castro AF, Feldman HI, Kusek JW, Eggers P, Lente FV, Greene T, Coresh J (2009) A new equation to estimate glomerular filtration rate. Ann Intern Med 150:604 612. https://doi.org/10.7326/0003-4819-150-9-200905050-00006

Lin YS, Lee WJ, Wang SJ, Fuh JL (2018) Levels of plasma neurofilament light chain and cognitive function in patients with Alzheimer or Parkinson disease. Sci Rep. https://doi.org/10.1038/ s41598-018-35766-w

Lu C-H, Macdonald-Wallis C, Gray E, Pearce N, Petzold A, Norgren N, Giovannoni G, Fratta P, Sidle K, Fish M, Orrell R, Howard R, Talbot K, Greensmith L, Kuhle J, Turner MR, Malaspina A (2015) Neurofilament light chain: a prognostic biomarker in amyotrophic lateral sclerosis. Neurology 84(22):2247-2257. https://doi.org/10. 1212/WNL.0000000000001642

Manouchehrinia A, Piehl F, Hillert J, Kuhle J, Alfredsson L, Olsson T, Kockum I (2020a) Confounding effect of blood volume and body mass index on blood neurofilament light chain levels. Annals of Clinical and Translational Neurology. https://doi.org/10.1002/ acn3.50972

Manouchehrinia A, Stridh P, Khademi M, Leppert D, Barro C, Michalak Z, Benkert P, Lycke J, Alfredsson L, Kappos L, Piehl F, Olsson T, Kuhle J, Kockum I (2020b) Plasma neurofilament light levels are associated with risk of disability in multiple sclerosis. Neurology. https://doi.org/10.1212/WNL.0000000000009571

Marques TM, Van Rumund A, Oeckl P, Kuiperij HB, Esselink RAJ, Bloem BR, Otto M, Verbeek MM (2019) Serum NFL discriminates Parkinson disease from atypical parkinsonisms. Neurology. https://doi.org/10.1212/WNL.0000000000007179

Mellgren A, Price RW, Hagberg L, Rosengren L, Brew BJ, Gisslén M (2007) Antiretroviral treatment reduces increased CSF neurofilament protein (NFL) in HIV-1 infection. Neurology 69(15):15361541. https://doi.org/10.1212/01.wnl.0000277635.05973.55

Novakova L, Zetterberg H, Sundström P, Axelsson M, Khademi M, Gunnarsson M, Malmeström C, Svenningsson A, Olsson T, Piehl F, Blennow K, Lycke J (2017) Monitoring disease activity in multiple sclerosis using serum neurofilament light protein. Neurology. https://doi.org/10.1212/WNL.0000000000004683

Olsson B, Portelius E, Cullen NC, Sandelius A, Zetterberg H, Andreasson U, Höglund K, Irwin DJ, Grossman M, Weintraub D, Chen-Plotkin A, Wolk D, McCluskey L, Elman L, Shaw LM, Toledo JB, McBride 
J, Hernandez-Con P, Lee VMY, Blennow K (2019) Association of Cerebrospinal Fluid Neurofilament Light Protein Levels with Cognition in Patients with Dementia, Motor Neuron Disease, and Movement Disorders. JAMA Neurol. https://doi.org/10.1001/jamaneurol. 2018.3746

Peterson J, Gisslen M, Zetterberg H, Fuchs D, Shacklett BL, Hagberg L, Yiannoutsos CT, Spudich SS, Price RW (2014) Cerebrospinal fluid (CSF) neuronal biomarkers across the spectrum of HIV infection: Hierarchy of injury and detection. PLoS One. https:// doi.org/10.1371/journal.pone.0116081

Yilmaz A, Blennow K, Hagberg L, Nilsson S, Price RW, Schouten J, Spudich S, Underwood J, Zetterberg H, Gisslén M (2017)
Neurofilament light chain protein as a marker of neuronal injury: review of its use in HIV-1 infection and reference values for HIV-negative controls. In Expert Review of Molecular Diagnostics 17(8)761-770. https://doi.org/10.1080/14737159.2017.13413 13

Zetterberg H (2016) Neurofilament light: a dynamic cross-disease fluid biomarker for neurodegeneration. In Neuron. https://doi.org/10. 1016/j.neuron.2016.06.030

Publisher's Note Springer Nature remains neutral with regard to jurisdictional claims in published maps and institutional affiliations.

\section{Authors and Affiliations}

\section{Jasmini Alagaratnam ${ }^{1,2}$ (D) - Davide De Francesco ${ }^{3}$. Henrik Zetterberg ${ }^{4,5,6,7}$. Amanda Heslegrave . Jamie Toombs $^{4}$. Neeltje A. Kootstra ${ }^{8}$. Jonathan Underwood ${ }^{1,9,10}$. Magnus Gisslen ${ }^{11,12} \cdot$ Peter Reiss ${ }^{13,14,15}$. Sarah Fidler ${ }^{1,2}$. Caroline A. Sabin ${ }^{3} \cdot$ Alan Winston ${ }^{1,2}$}

1 Department of Infectious Disease, Faculty of Medicine, Imperial College London, London, UK

2 Department of Genitourinary Medicine \&, HIV, St Mary's Hospital, Imperial College Healthcare NHS Trust, London, UK

3 Institute for Global Health, University College London, London, UK

4 UK Dementia Research Institute at University College London, London, UK

5 Department of Neurodegenerative Disease, UCL Institute of Neurology, Queen Square, University College London, London, UK

6 Department of Psychiatry and Neurochemistry, Institute of Neuroscience and Physiology, the Sahlgrenska Academy at the University of Gothenburg, Mölndal, Sweden

7 Clinical Neurochemistry Laboratory, Sahlgrenska University Hospital, Mölndal, Sweden

8 Department of Experimental Immunology, Amsterdam UMC, University of Amsterdam, Amsterdam, The Netherlands
9 Division of Infection and Immunity, Cardiff University, Cardiff, UK

10 Department of Infectious Diseases, Cardiff and Vale University Health Board, Cardiff, UK

11 Department of Infectious Diseases, Institute of Biomedicine, Sahlgrenska Academy, University of Gothenburg, Gothenburg, Sweden

12 Department of Infectious Diseases, Region Västra Götaland, Sahlgrenska University Hospital, Gothenburg, Sweden

13 Department of Global Health, Amsterdam University Medical Centers, University of Amsterdam, Amsterdam, The Netherlands

14 Amsterdam Institute for Global Health and Development, Amsterdam, The Netherlands

15 Stichting HIV Monitoring, Amsterdam, The Netherlands 